\title{
CORRECTION
}

\section{Correction to: Clinical predictors of mortality due to COVID-19 based on an analysis of data of 150 patients from Wuhan, China}

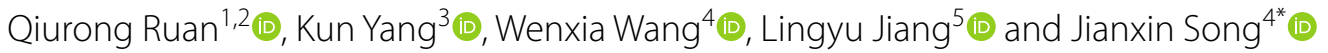

๑ 2020 Springer-Verlag GmbH Germany, part of Springer Nature

\section{Correction to: Intensive Care Med}

https://doi.org/10.1007/s00134-020-05991-x

The original version of this article unfortunately contained mistakes. There is an incorrect unit of IL- 6 in Fig. 1 and Supplementary Table 1 . It should be $\mathrm{pg} / \mathrm{mL}$ rather than $\mathrm{ng} / \mathrm{mL}$. The corrected Fig. 1 and Supplementary Table 1 can be found below. The authors apologize for the mistakes.

Full author information is available at the end of the article 


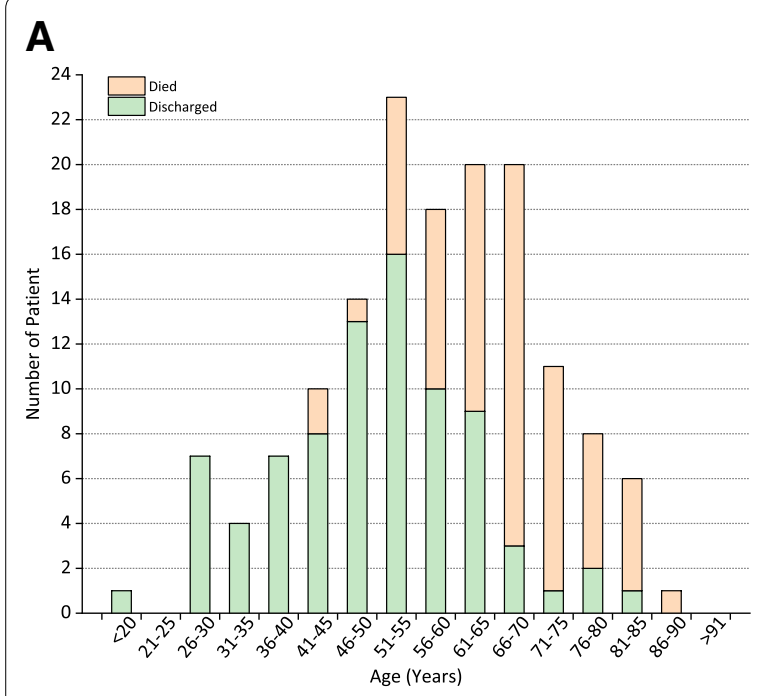

C

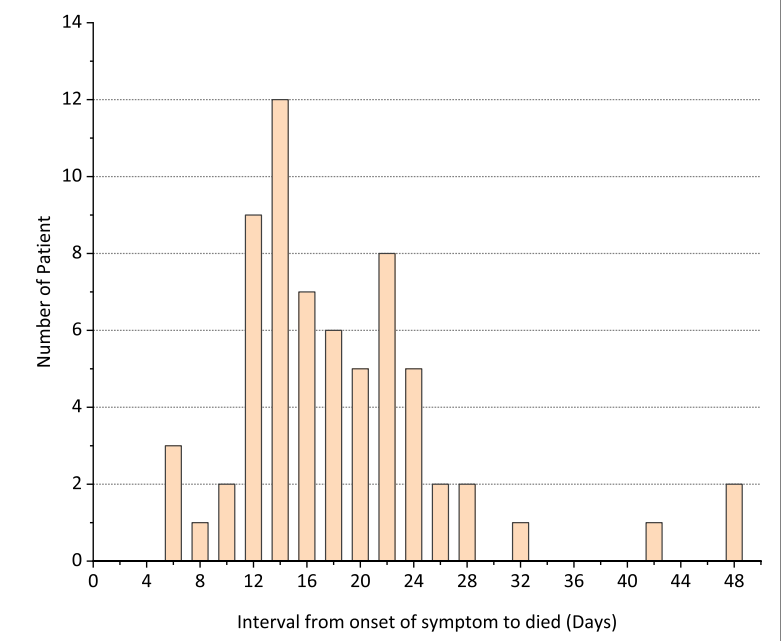

B $\square$ Discharged

D
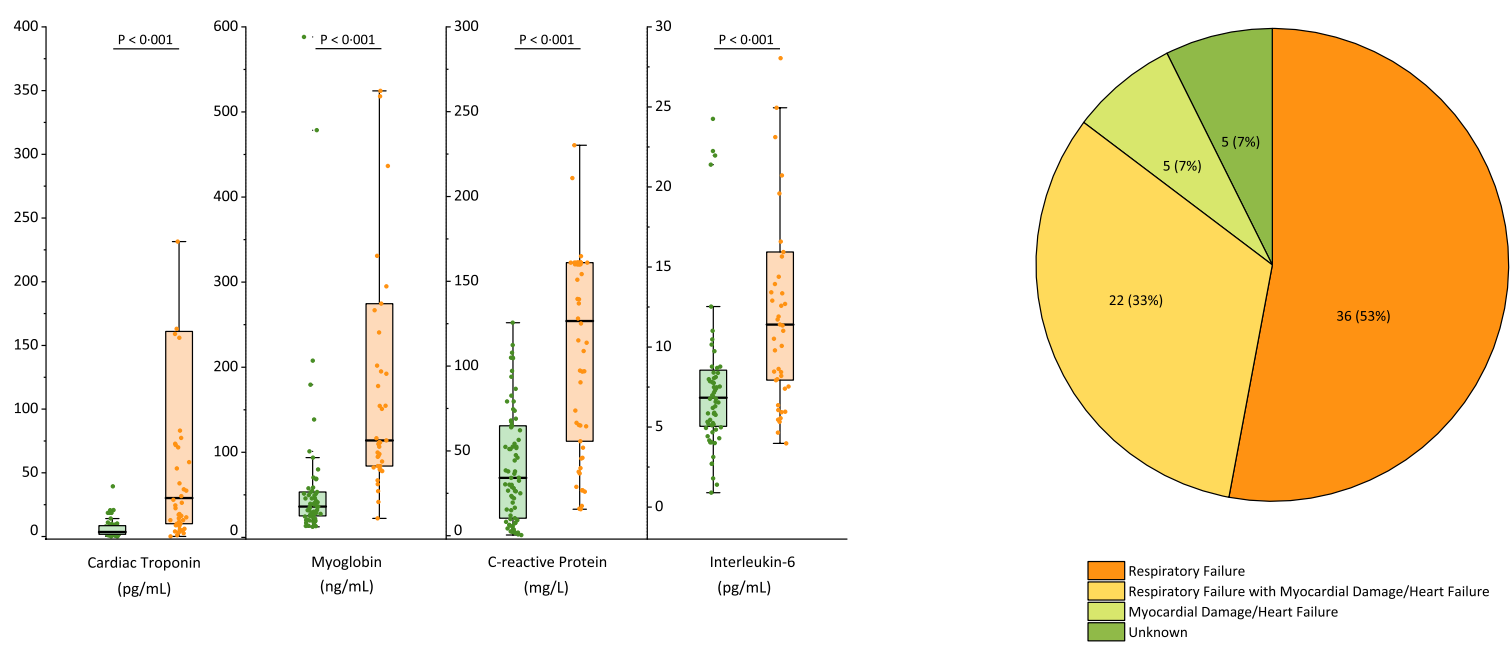

Fig. 1 a Age distribution of patients with confirmed COVID-19; b key laboratory parameters for the outcomes of patients with confirmed COVID19; c interval from onset of symptom to death of patients with confirmed COVID-19; $\mathbf{d}$ summary of the cause of death of 68 died patients with confirmed COVID-19 
Supplementary Table 1: Summary of clinical features and laboratory results of the patients with confirmed COVID-19

\begin{tabular}{|c|c|c|c|c|}
\hline & $\begin{array}{l}\text { Normal } \\
\text { Range }\end{array}$ & $\begin{array}{l}\text { Died } \\
(N=68)\end{array}$ & $\begin{array}{l}\text { Discharged } \\
(\mathrm{N}=\mathbf{8 2})\end{array}$ & p-value \\
\hline \multicolumn{5}{|l|}{ Demographics } \\
\hline Age, years & & $67(15-81)$ & $50(44-81)$ & $<0.001$ \\
\hline \multicolumn{5}{|l|}{ Sex } \\
\hline Male & & $49(72 \%)$ & $53(65 \%)$ & \\
\hline Female & & $19(28 \%)$ & $29(35 \%)$ & \\
\hline Comorbidities & & $43(63 \%)$ & $34(41 \%)$ & 0.01 \\
\hline Hypertension & & $29(43 \%)$ & $23(28 \%)$ & 0.07 \\
\hline Cardiovascular disease & & $13(19 \%)$ & 0 & $<0.001$ \\
\hline Diabetes & & $12(18 \%)$ & $13(16 \%)$ & 0.88 \\
\hline Cerebrovascular disease & & $7(10 \%)$ & $5(6 \%)$ & 0.49 \\
\hline Chronic obstructive pulmonary disease & & $2(3 \%)$ & $1(1 \%)$ & 0.59 \\
\hline Chronic kidney disease & & $2(3 \%)$ & 0 & 0.20 \\
\hline Malignancy & & $2(3 \%)$ & $1(1 \%)$ & 0.59 \\
\hline Chronic liver disease & & $1(1 \%)$ & $3(4 \%)$ & 0.63 \\
\hline Connective tissue disease & & $1(1 \%)$ & 0 & 0.45 \\
\hline \multicolumn{5}{|l|}{ Signs and Symptoms } \\
\hline Fever & & $59(87 \%)$ & $68(83 \%)$ & 0.67 \\
\hline Cough & & $51(75 \%)$ & $59(72 \%)$ & 0.81 \\
\hline Sputum production & & $29(43 \%)$ & $25(30 \%)$ & 0.17 \\
\hline Fatigue & & $15(22 \%)$ & $22(27 \%)$ & 0.63 \\
\hline Myalgia & & $9(13 \%)$ & $10(12 \%)$ & 1 \\
\hline Haemoptysis & & $3(4 \%)$ & 0 & 0.09 \\
\hline Dyspnea & & $59(87 \%)$ & $51(62 \%)$ & 0.001 \\
\hline Respiratory failure & & $58(85 \%)$ & $13(16 \%)$ & $<0.001$ \\
\hline ARDS & & $55(81 \%)$ & $7(9 \%)$ & $<0.001$ \\
\hline Acute kidney injury & & $21(31 \%)$ & $2(2 \%)$ & $<0.001$ \\
\hline Infection & & $11(16 \%)$ & $1(1 \%)$ & 0.002 \\
\hline \multicolumn{5}{|l|}{ Treatments } \\
\hline ICU admission & & $30(44 \%)$ & $11(13 \%)$ & $<0.001$ \\
\hline High flow nasal cannula & & $31(46 \%)$ & $10(12 \%)$ & $<0.001$ \\
\hline Non-invasive mechanical ventilation & & $46(68 \%)$ & $5(6 \%)$ & $<0.001$ \\
\hline Invasive mechanical ventilation & & $25(37 \%)$ & 0 & $<0.001$ \\
\hline ECMO & & $7(10 \%)$ & 0 & 0.003 \\
\hline CRRT & & $5(7 \%)$ & 0 & 0.02 \\
\hline Prone position mechanical ventilation & & $3(4 \%)$ & 0 & 0.09 \\
\hline Antiviral treatment & & $33(49 \%)$ & $55(67 \%)$ & 0.05 \\
\hline Antibiotic treatment & & $63(93 \%)$ & $80(98 \%)$ & 0.66 \\
\hline Antifungal treatment & & $12(18 \%)$ & $5(6 \%)$ & 0.04 \\
\hline Glucocorticoid treatment & & $31(46 \%)$ & $22(27 \%)$ & 0.02 \\
\hline Time from onset of symptom to start of Glucocorticoid treatment, days & & $11.7(6.9)$ & $11.4(4.4)$ & 0.89 \\
\hline Time from hospital admission to start of Glucocorticoid treatment, days & & $1.5(1.2)$ & $3.2(2.7)$ & 0.01 \\
\hline Glucocorticoid treatment duration, days & & $7.2(6.7)$ & $7.0(5.0)$ & 0.92 \\
\hline Supportive therapy & & $45(66 \%)$ & $18(22 \%)$ & $<0.001$ \\
\hline Length of stay, days & & $7.4(5.5)$ & $12.3(4.4)$ & $<0.001$ \\
\hline \multicolumn{5}{|l|}{ Laboratory Findings } \\
\hline Time from onset of symptom to test, days & & $11.6(6.8)$ & $9.8(4.3)$ & 0.07 \\
\hline White blood cell count, $\times 10^{9} / \mathrm{L}$ & $3.50-9.50$ & $10.62(4.76)$ & $6.76(3.49)$ & $<0.001$ \\
\hline Lymphocyte count, $\times 10^{9} / \mathrm{L}$ & $1.10-3.20$ & $0.60(0.32)$ & $1.42(2.14)$ & $<0.001$ \\
\hline
\end{tabular}




\begin{tabular}{|c|c|c|c|c|}
\hline & $\begin{array}{l}\text { Normal } \\
\text { Range }\end{array}$ & $\begin{array}{l}\text { Died } \\
(N=68)\end{array}$ & $\begin{array}{l}\text { Discharged } \\
(\mathrm{N}=82)\end{array}$ & p-value \\
\hline Haemoglobin, g/L & $130.0-175.0$ & $127.0(16.7)$ & $127.6(16.3)$ & 0.82 \\
\hline Platelet count, $\times 10^{9} / \mathrm{L}$ & $125.0-350.0$ & $173.6(67.7)$ & $222.1(78.0)$ & $<0.001$ \\
\hline Albumin, g/L & $35.0-52.0$ & $28.8(3.8)$ & $32.7(3.8)$ & $<0.001$ \\
\hline Alanine aminotransferase, $\mathrm{U} / \mathrm{L}$ & $9.0-50.0$ & $170.8(991.6)$ & $48.68(83.1)$ & 0.35 \\
\hline Aspartate aminotransferase, $\mathrm{U} / \mathrm{L}$ & $15.0-40.0$ & $288.9(1875.5)$ & $40.7(57.8)$ & 0.31 \\
\hline Total bilirubin, $\mu \mathrm{mol} / \mathrm{L}$ & $0.0-26.0$ & $18.1(10.7)$ & $12.8(6.8)$ & 0.001 \\
\hline Blood urea nitrogen, $\mathrm{mmol} / \mathrm{L}$ & $3.1-8.0$ & $8.65(4.5)$ & $5.11(2.1)$ & $<0.001$ \\
\hline Creatinine, umol/L & $59.0-104.0$ & $91.2(56.2)$ & $72.1(22.2)$ & 0.02 \\
\hline Creatine kinase, U/L & $50.0-310.0$ & $319.4(838.5)$ & $231.7(862.3)$ & 0.56 \\
\hline Lactate dehydrogenase, U/L & $120.0-250.0$ & $905.8(2619.1)$ & $297.9(110.4)$ & 0.08 \\
\hline Cardiac troponin, pg/mL & $2.0-28.0$ & $30.3(151.0)$ & $3.5(6.2)$ & $<0.001$ \\
\hline Myoglobin, ng/mL & $0.0-146.9$ & $258.9(307.6)$ & $77.7(136.1)$ & $<0.001$ \\
\hline C-reactive protein, mg/L & $0.0-5.0$ & $126.6(106.3)$ & $34.1(54.5)$ & $<0.001$ \\
\hline Interleukin-6, pg/mL & $0.0-7.0$ & $11.4(8.5)$ & $6.8(3.61)$ & $<0.001$ \\
\hline Serum ferritin, $\mathrm{ng} / \mathrm{mL}$ & $21.8-274.7$ & $1297.6(1030.9)$ & $614.0(752.2)$ & $<0.001$ \\
\hline
\end{tabular}

Data are $\mathrm{n}(\%), \mathrm{n} / \mathrm{N}(\%)$, mean (SD), and median (IQR). When the data were normally distributed, continuous variables were then described using median and interquartile range (IQR) values.

$\mathrm{ARDS}=$ acute respiratory distress syndrome. $\mathrm{ECMO}=$ extracorporeal membrane oxygenation. $\mathrm{ICU}=$ intensive care unit. $\mathrm{CRRT}=$ continuous renal replacement therapy.

\section{Electronic supplementary materia}

The online version of this article (https://doi.org/10.1007/s00134-020-06028-z) contains supplementary material, which is available to authorized users.

\section{Author details}

${ }^{1}$ Institute of Pathology, Tongji Hospital, Tongji Medical College, Huazhong University of Science and Technology, 1095 Jiefang Avenue, Wuhan 430030, Hubei, China. ${ }^{2}$ Department of Pathology, School of Basic Medicine, Tongji Medical College, Huazhong University of Science and Technology, 1095 Jiefang Avenue, Wuhan 430030, Hubei, China. ${ }^{3}$ Department of Dermatology, Tongji Hospital, Tongji Medical College, Huazhong University of Science and Technology, 1095 Jiefang Avenue, Wuhan 430030, Hubei, China. ${ }^{4}$ Department of Infectious Diseases, Tongji Hospital, Tongji Medical College, Huazhong
University of Science and Technology, 1095 Jiefang Avenue, Wuhan 430030, Hubei, China. ${ }^{5}$ Department of Clinical Immunology, Tongji Hospital, Tongji Medical College, Huazhong University of Science and Technology, 1095 Jiefang Avenue, Wuhan 430030, Hubei, China.

\section{Publisher's Note}

Springer Nature remains neutral with regard to jurisdictional claims in published maps and institutional affiliations.

Published online: 6 April 2020 